\title{
Glass transition in a two-dimensional system of magnetic colloids
}

\author{
H. König*, K. Zahn ${ }^{\dagger}$ and G. Maret* \\ ${ }^{*}$ University of Konstanz, Department of Physics, 78457 Konstanz, Germany \\ †present address: Ascom Systec Ltd., 5504 Mägenwil, Switzerland
}

\begin{abstract}
We describe experiments on binary mixtures of superparamagnetic colloidal particles confined by gravity to a flat horizontal water-air interface. The colloids repel each other because of their magnetic dipole moments induced by a vertical external magnetic field $B$. By tuning $B$, the effective temperature of the system can be adjusted over several orders of magnitude. Particle coordinates are monitored by video-microscopy over more than five decades in time. Measured radial pairdistribution functions $g(r)$ and mean-square displacements illustrate that this system is an ideal model of a two-dimensional (2D) glass former. We find that the effects of small amounts of aggregated particles only weakly affect the averaged structure and dynamics. Locally, a small number of elementary structural elements are observed each characterized by a special triangular shape. These triangles arrange in dense mostly space-filling arrays and account for the essential features of $g(r)$. The long-time $\alpha$-relaxation is related to drifts of arrays as well as erosion due to single particle and collective hopping events.
\end{abstract}

Glasses are solids without structural long-range order and with dynamics frozen-in at least at time scales of typical experiments [1]. One of the most striking and yet unexplained phenomenon in glass physics is the widely universal slowing down of structural relaxations when approaching the glass transition from the liquid side. Physical quantities as different as viscosity, frequency dependent dielectric constants or density correlations obtained from inelastic neutron scattering behave very similar in many different atomic, molecular or macromolecular systems [2]. Recent experiments on colloidal glasses [3, 4] as well as computer simulations [5, 6, 7] suggest that essential properties of glass formers can be understood by studying simple model systems. Both techniques provide time dependent particle coordinates opening a new way to investigate the microscopic structural properties of glasses and their influence on local dynamic relaxations near the glass transition. While simulations have dealt with three dimensional and two dimensional systems, we are not aware of any experiment on true two-dimensional colloidal glasses.

In this paper, we describe an experimental model system which shows all features of a glass former in 2D [8]. Binary mixtures of superparamagnetic colloidal particles are confined by gravity to a horizontal water-air interface in a flat hanging drop geometry. The interparticle magnetic dipole interaction is controlled by a magnetic field applied vertically in order to adjust the effective system temperature. The interaction strength is precisely calibrated. Time-dependent particle positions are determined by video-microscopy and partial radial pair-distribution functions $g_{i j}(r)$ and mean-square displacements $\left\langle\Delta r^{2}(t)\right\rangle=\left\langle\left(\vec{r}_{i}(0)-\vec{r}_{i}(t)\right)^{2}\right\rangle$ were calculated. The shapes of these functions do not significantly depend on small amounts of aggregates and compare surprisingly well with those of $3 \mathrm{D}$ glass formers $[9,10]$. Locally, a small number of elementary structural elements of hexagonal, squared body-centered, and 10-fold symmetry are observed. They arrange in dense mostly spacefilling arrays and account for the essential features of $g_{i j}(r)[11,12]$.

A. Experimental set-up: The commercial [13] monodisperse colloidal PMMA particles used are porous and doped with magnetite nanocrystals making them superparamagnetic. The big $(b)$ (Dynabeads M-450, uncoated) and small ( $s$ ) (dried Dynabeads M280 uncoated) particles have diameters $d_{b}=4.7 \mu \mathrm{m}$ and $d_{s}=2.8 \mu \mathrm{m}$, and densities $\rho_{b} \approx 1.5 \cdot 10^{3} \mathrm{~kg} / \mathrm{m}^{3}$ and $\rho_{s} \approx 1.3 \cdot 10^{3} \mathrm{~kg} / \mathrm{m}^{3}$, respectively. Their magnetic susceptibilities were determined by comparison $g(r)$ measured in the liquid state with Browniandynamics simulations [14]. We thus obtained low field magnetic susceptibilities $\chi_{b}=6.2 \cdot 10^{-11} A m^{2} / T$ and $\chi_{s}=6.6 \cdot 10^{-12} A m^{2} / T . \chi_{b}$ was found constant within the range of $B$-values used ( 0 to $\approx 4 m T$ ), while $\chi_{s}$ lowers due to magnetic saturation above $3 m T$ reaching about $80 \%$ of the low- $B$-value at $4 m T$. These values agree with measurements of magnetophoretic mobilities of individual particles and direct SQUID-magnetometry [15]. In order to prevent aggregation, the big Dynabeads were 
stabilized by sodium dodecyl sulfonate (SDS) while the small Dynabeads were found to be stable in pure water.

The colloidal suspension was filled into the cylindrical holes of a glass cell as sketched in Fig. 1. The larger hole was $8 \mathrm{~mm}$ in diameter and $1 \mathrm{~mm}$ in height. The cell was mounted upside down in the sample holder which was temperature stabilized by a large copper block. The liquid drop was suspended by interfacial tension, that is by pinning the water-air interface at the edge of the cylindrical cell due to hydrophobic treatment of the outer cell surface. The colloids sediment on the interface and form a monolayer with average density dependent on the amount of colloids present in the initial bulk suspension. The curvature of the drop was adjusted by tuning the water volume through a computer controlled nano-syringe. In order to minimize convection due to filling, the volume of the large cell was adjusted by adding/removing water in the small satellite cell connected to the main cell by a thin capillary. The interface was kept flat by observation of the sharpness of particle images everywhere in the focal plane of a 40x microscope lens. Thus a flatness of $<1 \mu \mathrm{m}$ at a cell diameter of $8 \mathrm{~mm}$ was obtained. Additionally, the horizontal orientation of the liquid-air interface was controlled through adjustment of the optical table to $\pm 10^{-4} \mathrm{rad}$.

The system can be considered almost ideally twodimensional: First, the thermal gravitational length of the big and small colloids is about $8 \mathrm{~nm}$ and $62 \mathrm{~nm}$, respectively, much smaller than both particle diameters. Secondly, the gravitational force of the colloids is essentially unable to bulge the surface against surface tension. Finally, vertical particle displacements due to thermal capillary waves are less than $\sim 1 \mathrm{~nm}$ and, thus, can be neglected [16]. In order to reduce collective particle drifts induced by convective flow of water, thermal gradients and disturbances of the water-air interface were suppressed by surrounding the entire experimental setup by a polystyrene box and air conditioning of the laboratory. Well equilibrated samples could be conserved for several weeks.

A homogeneous magnetic field, $B$, was applied perpendicular to the water-air interface by coils inducing vertical magnetic moments $\vec{M}=\chi \cdot \vec{B}$ in the colloidal particles. The magnetic field generated by the induced magnetic moments themselves is only of the order of $1 \%$ of $B$ (at maximum concentration) and therefore can be neglected. Hence, two induced magnetic moments interact by a repulsive dipole potential proportional to $\chi^{2} B^{2} / r^{3}$, where $r$ is the center-to-center distance between two colloids. Other contributions to the interaction potential can be neglected [14].

The interaction strength is characterized by an interaction parameter, $\Gamma$, defined by the ratio of the magnetic interaction potential $E_{\text {magn }}(B)$ to the thermal energy $k_{B} T$. $\Gamma$ corresponds to an inverse system temperature, $T_{\text {sys }}$ tun-

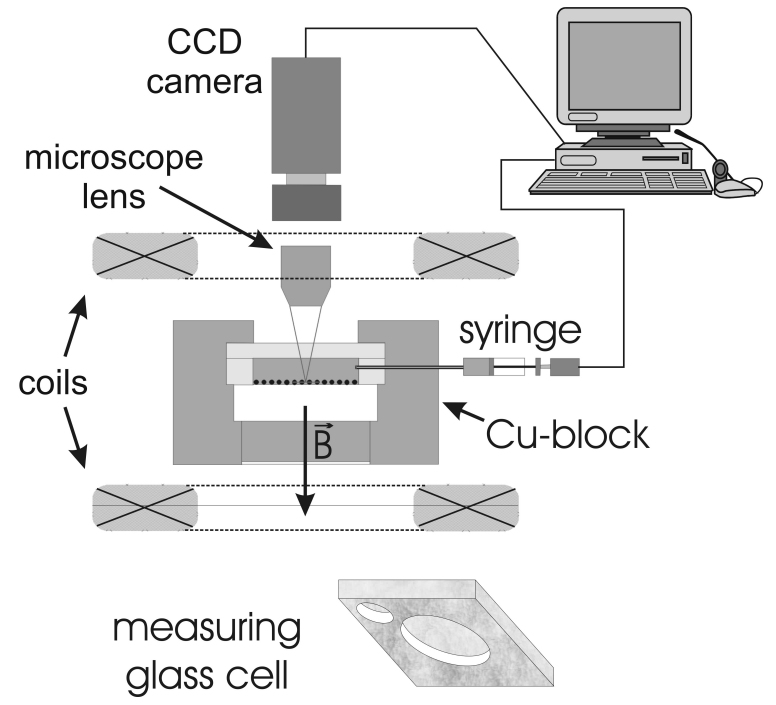

FIGURE 1. Sketch of the experimental set-up (see text for details).

able by $B$. With the particle area density, $\rho$, corresponding to the averaged particle distance $\bar{r}=\rho^{-1 / 2}$, and the number ratio $\xi$ of the small to all particles the $\Gamma$-value of the binary $2 \mathrm{D}$ colloidal mixture can be defined as:

$\Gamma=\frac{E_{\text {magn }}}{k_{B} T}=\frac{\mu_{0}}{4 \pi} \frac{B^{2}(\rho \pi)^{3 / 2}}{k_{B} T}\left(\xi \cdot \chi_{s}+(1-\xi) \cdot \chi_{b}\right)^{2}$

$\rho$ and $T$ remain constant during an experiment, thus $T_{\text {sys }}$ decreases for increasing $\Gamma$.

Typically 1,000 particles out of approximately 100,000 in the cell were observed by video-microscopy, at locations far away from the cell edge, in order to avoid effects of the cell boundaries. Time-dependent positions of particles were determined in real-time by digitization on a frame grabber card and image processing on a PC. Every $\approx 0.3 s$ a complete set of uniquely labelled particle coordinates were determined depending on the number of observed particles and saved for evaluation later on: Each video frame was converted into a binary image using a brightness cut-off value such that colloids appeared as black discs on white background. The center of mass of a disc's area gives the particle position which was calculated with an accuracy better than $1 \mu \mathrm{m}$. The disc's area indicated whether a given particle was a small or a big one.

For purposes of more efficient data storage, and in order to display the mean-square displacements equidistant on a logarithmic time-scale over many decades, a multiple- $\tau$-algorithm was used. In this algorithm the sampling time $\Delta t$ is doubled after every 1,000 sampling intervals. The initial maximum sampling rate is used for 

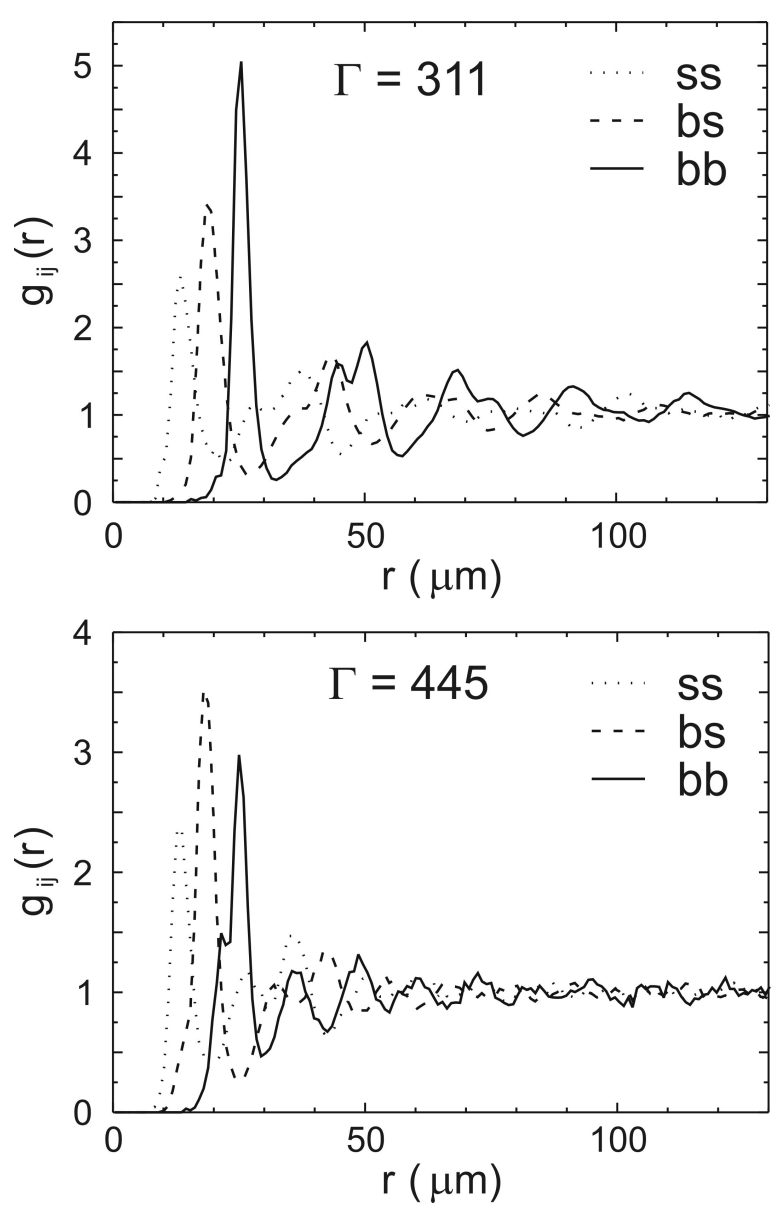

FIGURE 2. Partial radial pair-distribution functions $g_{i j}(r)$, $g_{b s}(r)$ and $g_{s s}(r)$ for a sample with a low number of aggregates $\left(\Gamma=311, \xi=0.29, \rho=1.78 \cdot 10^{-3} \mu m^{-2}\right)$ and for a sample with a high number of aggregates $(\Gamma=445, \xi=0.57, \rho=$ $\left.2.08 \cdot 10^{-3} \mu \mathrm{m}^{-2}\right)$.

data processing in order to enable a reliable identification of particles between consecutive time steps.

Some uncertainties in the trajectories originate either from drift effects caused by convective flow of water or from aggregates of a few colloids (mostly two small or one small and one big particle) having different magnetic susceptibilities and different diffusion coefficients than isolated particles. Although the drift was typically smaller than $1 \mu \mathrm{m} / \mathrm{h}$, we minimized this effect by subtracting the average displacement of all particles from the displacement of each particle. The overall stability of the system itself was sufficiently good to obtain reliable data up to more than $10^{5} \mathrm{~s}$.

B. Structure: Partial pair-distribution functions $g_{i j}(r)$ for the different pairs $i j$ of big and small colloids were obtained by spatial averaging over typically about 100 independent particle configurations. Sufficient ensemble averaging was obtained by additional time averaging. In
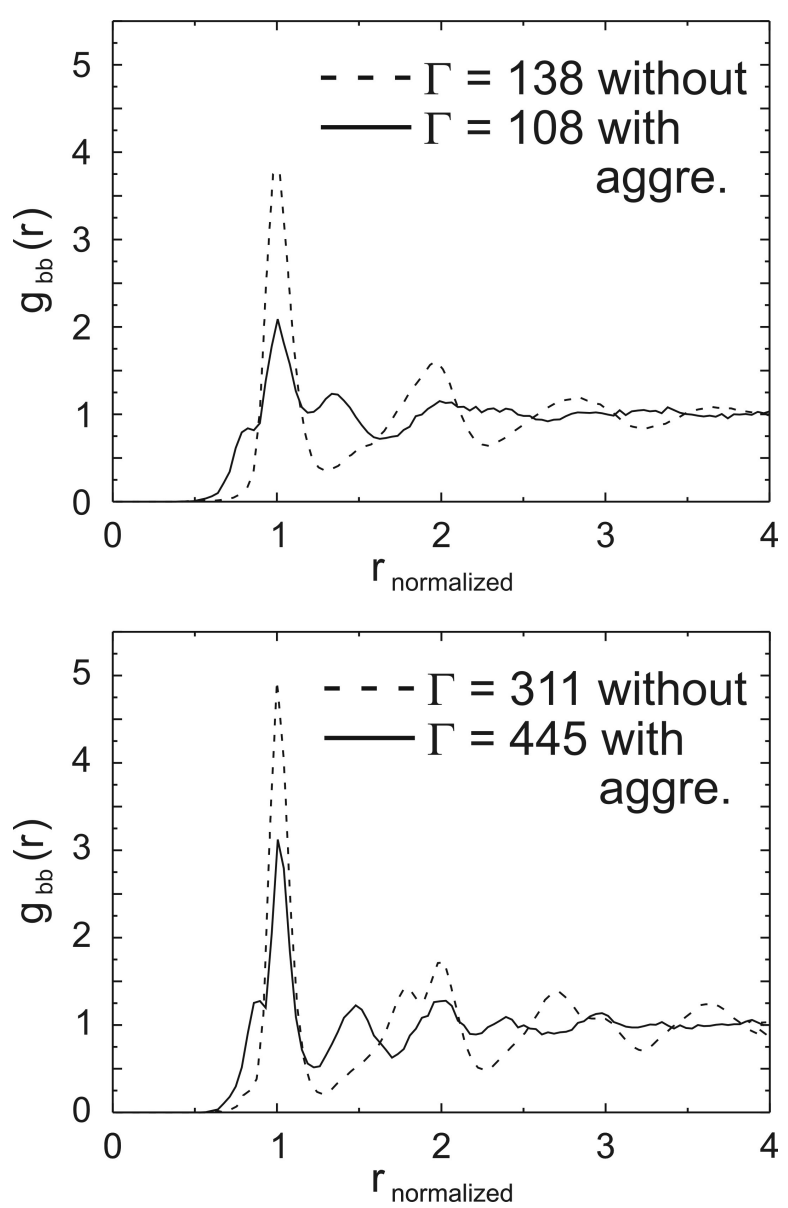

FIGURE 3. Comparison of big/big radial pair-distribution functions $g_{b b}(r)$ for low (a) and for high (b) $\Gamma$-values. Dashed curves correspond to a sample with few $(<10)$ aggregates, continuous lines refer to a sample with $(\approx 50)$ explored aggregates. $r$-values are normalized to the average interparticle distance $r_{b b}$ obtained from the positions of the biggest peak of $g_{b b}(r)$.

Fig. $2 g_{i j}(r)$ for the three pairs $(b b),(b s)$ and $(s s)$ are compared for two different samples. The highest peaks correspond to the most frequent interparticle distances which are rather sharp. A series of additional peaks at higher distances are observed for all three partial $g_{i j}(r)$ indicating substantial local order of the binary mixture. With increasing $\Gamma$ the peaks of $g_{i j}(r)$ become sharper and narrower and more features appear at higher distances, in particular a splitting of the second maximum. These observations are well-known "finger prints" of amorphous structures of glass formers.

Fig. 3 shows $g_{b b}(r)$ for two binary suspensions, one containing very few aggregates within the picture explored, while the other contains about 5 times more aggregates. For the sample containing more aggregates additional peaks in $g_{b b}(r)$, in particular at distances shorter than the average distance between big particles are seen. 


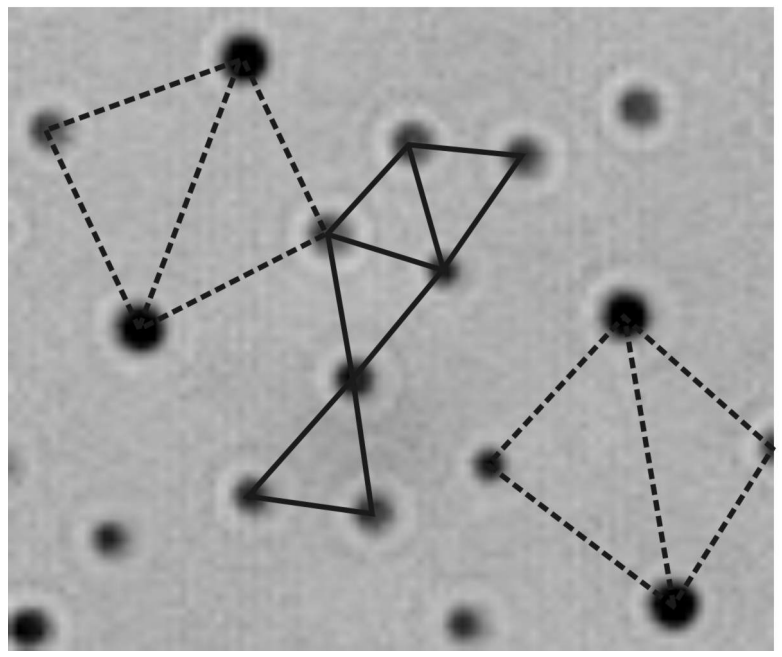

FIGURE 4. Hexagonal arrangements of small particles (solid lines) and rectangular triangles of one small and two big colloids (dashed lines).

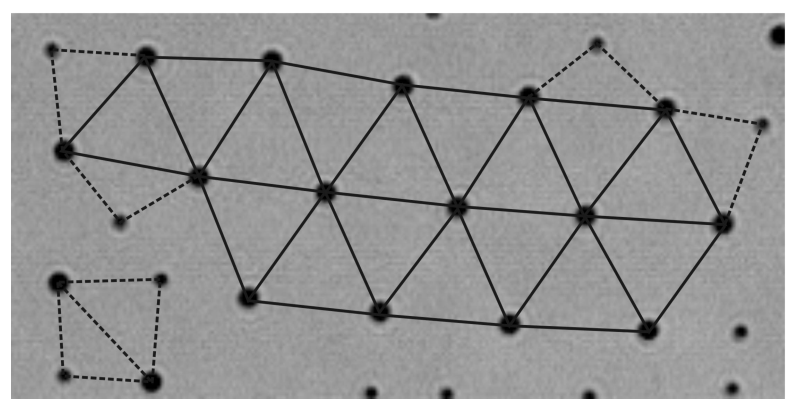

FIGURE 5. Hexagonal arrangements of big particles (solid lines) and rectangular triangles of one small and two big colloids (dashed lines).

Visual inspection of the pictures reveal that these shorter distances mostly originate from aggregates of two or three small particles (sticking together at mutual surface contact) which are processed by the particle recognition algorithm as one big particle. This observation indicates that $g(r)$ is rather sensitive to aggregates of this type. Note that, in contrast, the relaxation dynamics discussed below turns out rather insensitive to these aggregates.

The observed features in $g_{i j}(r)$ are related to highly ordered local arrangements of particles [12] which can be directly seen by inspection of local configurations. A few typical examples are given in the series of pictures Fig. 4 to Fig. 8 which were taken on different spots of the same sample for $\Gamma=411$. Such structures are disturbed by thermal particle fluctuations. Because of the purely repulsive dipole interaction it seems natural to expect local hexagonal packings in cases were only one species of particles happens to be around, Fig. 4

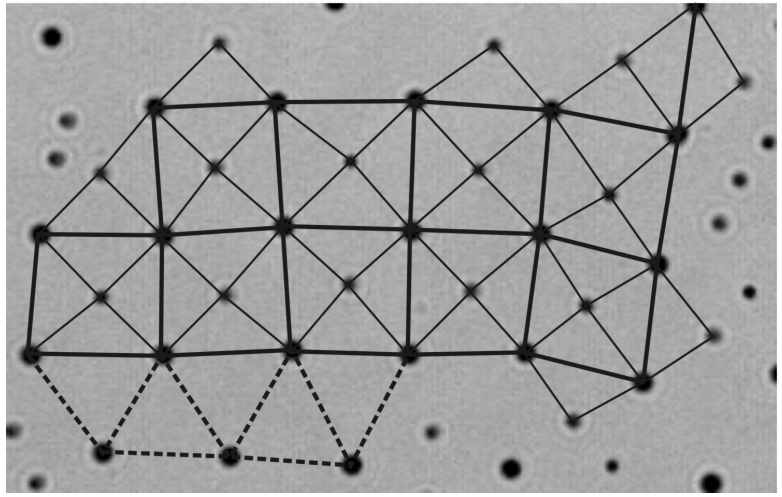

FIGURE 6. Quadratic arrangements of equal numbers of big and small particles (solid lines) and neighboring hexagonal triangles of only big colloids (dashed lines).

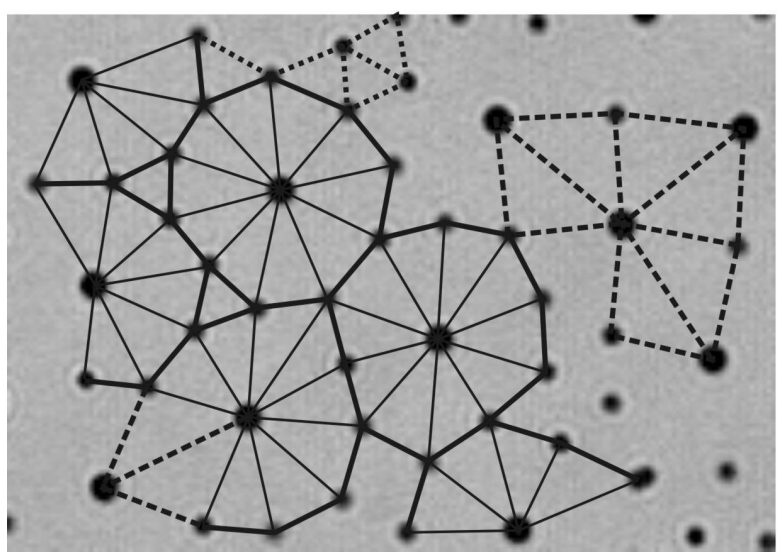

FIGURE 7. Arrangements of big particles surrounded by many small particles (solid lines). A configuration frequently observed consists of 10 small particles forming a ring-like structure around a big particle. Additionally, rectangular triangles of one small and two big colloids (dashed lines) and hexagonal triangles of only small particles (dotted lines) are depicted.

and Fig. 5. At locations of equal numbers of small and big particles a square body-centered lattice decorated like a checkerboard pattern is observed. Packings at high local densities of small particles are shown in Fig. 7 and Fig. 8. Because of the much smaller repulsion between two small particles as compared to the cases small/big and big/big, small particles get to substantially shorter mutual distances. This seems to stabilize lozenge-type configurations consisting of two big particles at the small angles of the lozenges and two small particles at the big angles, respectively. Often, 10 small particles are observed in a ring-like configuration around one big particle. Then, the small/big internal angle of the lozenge is close to $36 / 144$ degrees, respectively. This type of lozenge is also the smaller one of the two tiles required 

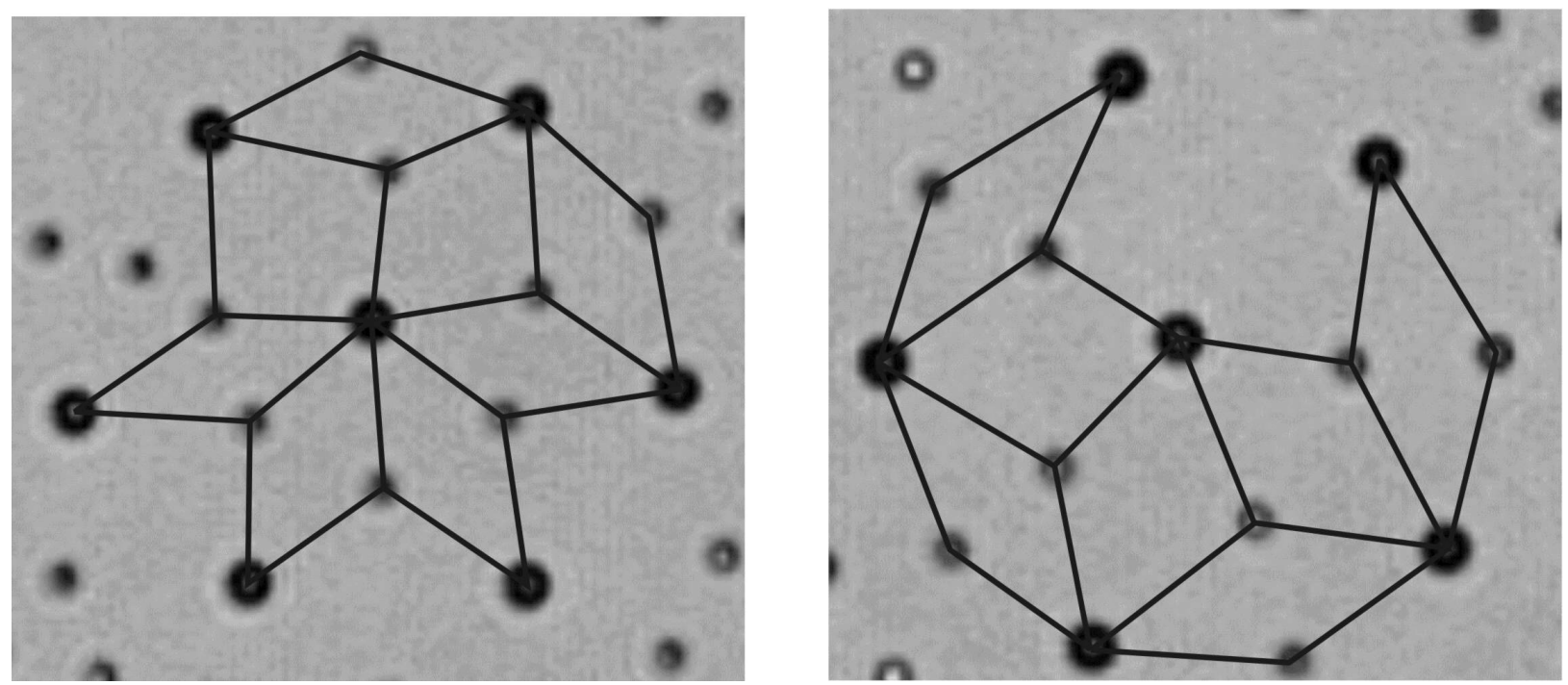

FIGURE 8. Local configurations revealing some lozenges close to Penrose tiles with small angles of 36 or 72 degrees at the big colloid $(\Gamma=411)$.

to obtain a quasi-crystal with Penrose tiling. At some locations the two Penrose tiles seem to appear, see Fig. 8. Similar observations are reported in [17].

Different local-ordered elementary particle arrangements coexist in direct neighborhood. These observations suggest that the amorphous structure of our 2D system is built up of different local structures which depend on local concentration and on local composition. The highly ordered local structures essentially account for all peaks observed in the partial radial pair distribution functions [12]. In addition, they give rise to significant features in the 3-point and 4-point correlation functions $[11,12]$

C. Dynamics: The overall dynamics of our system was studied in real space by mean-square displacements of individual particles, e.g. the time-dependent particle deviations from their initial positions: $\left\langle\Delta r^{2}(t)\right\rangle=$ $\left\langle\left(\vec{r}_{i}(0)-\vec{r}_{i}(t)\right)^{2}\right\rangle$. The mean-square displacements show a short-time behavior which is independent of $\Gamma$. In the time-range up to about $2 s$, the particles obey a diffusive motion with a diffusion constant somewhat smaller than the free Stokes diffusion due to the hydrodynamic interaction with the liquid-air interface [14]. For longer timescales and increasing $\Gamma$, the slope of $\left\langle\Delta r^{2}(t)\right\rangle$ diminishes, and a increasingly pronounced plateau appears. As in atomic or molecular glasses this plateau may be attributed to a so-called cage-effect. A particle stays in the cage of its neighbors until structural $\alpha$-relaxations allow it to escape. The long-time $\alpha$-relaxations cause an additional increase of the mean-square displacements at long times. An example is shown in Fig. 9.

It can be seen that short-time diffusion, plateau region and long-time diffusion are essentially insensitive to the

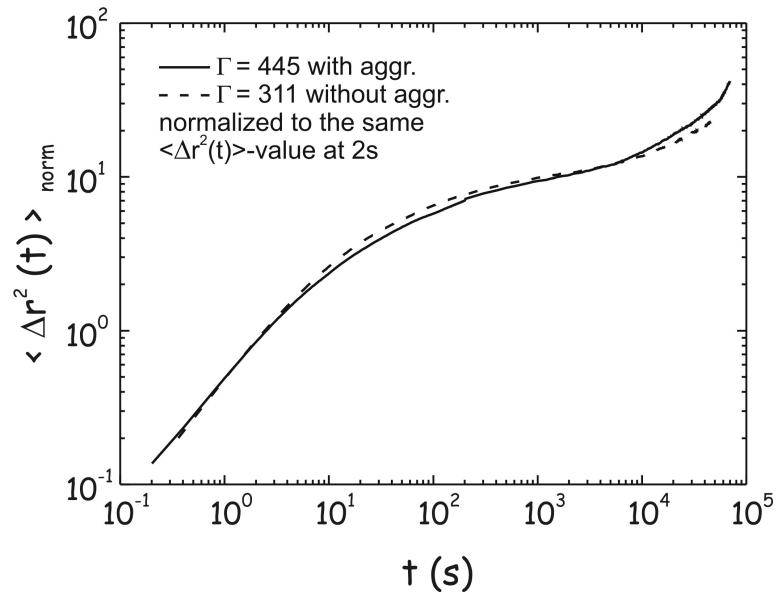

FIGURE 9. Mean square displacements of the two samples shown in Fig. 2. Because the two samples possessed different particle densities, e.g. different particle distances, the $\left\langle\Delta r^{2}(t)\right\rangle$ of the sample with $\Gamma=445$ was brought into line with the shorttime behavior of the other sample at $t=2 \mathrm{~s}$.

presence of some aggregates. This argues that the details of the local structure do not really matter for the relaxation dynamics and may suggest that other more polydisperse systems may relax in a qualitatively similar way.

Fig. 10 shows particle trajectories of a sample at rather high $\Gamma$. These data clearly reveal a strongly heterogeneous relaxation behavior: There are regions were particle motions are essentially restricted to diffusive motion around a given position, both for small and big particles. Some of these areas are seen to obey slow convective 


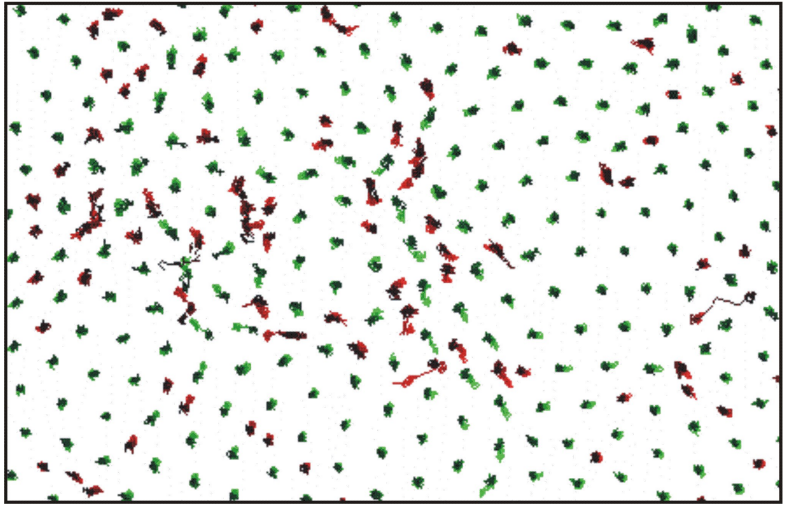

FIGURE 10. Trajectory plot of a part of the sample with $\Gamma=311$ of Fig. 2 monitored over a time interval of 34450 s for time steps between two data files of $\sim 252 s$. The color of the trajectory becomes darker as time goes on. Green: big particles, red: small particles.

drifts without much changes of the relative particle positions. Finally some particles have excursions much larger than the average diffusive displacement, sometimes even larger than the average interparticle distance. These particles have clearly left their cages, sometimes as isolated particles, sometimes as groups of two or more particles in a string-like motion. Thus the relaxation dynamics of this system is clearly quite heterogeneous. Slow collective drifts and single or multiple hopping events out of cages coexist. In a forthcoming study [12] a correlation between the locally ordered viz. disordered structures with the collective viz. single hopping dynamics will be discussed.

D. Conclusions: 2D binary colloidal suspensions with knob-tunable magnetic interparticle interactions are ideal systems to study structural and dynamic processes near the glass transition. The local structure remains liquidlike with more pronounced short-range order for decreasing $T_{s y s}$. Correspondingly, the particle dynamics slows down, as seen by an increased cage-effect. Regular elementary structures (such as triangular, square or rhombic particle arrangements) are clearly observed, presumably much easier to detect than equivalent clusters in 3D.

\section{ACKNOWLEDGMENTS}

This work was supported by the Deutsche Forschungsgemeinschaft, in the frame of SFB 513, project B6.

\section{REFERENCES}

1. Ediger, M. D., Angell, C. A., and Nagel, S. R., J. Chem. Phys. 100, 13200-13212 (1996)
2. Vilgis, T. A., "Models for Transport and Relaxation in Glass Forming and Complex Fluids: Universality?", in Disorder Effects on Relaxational Proceses, Glasses, Polymers, Proteins, edited by R. Richert, and A. Blumen, Springer-Verlag, Berlin, 1994, pp. 153-191.

3. Weeks, E. W., Crocker, J. C., Levitt, A. C., Schofield, A., and Weitz, D. A., Science 287, 627-631 (2000).

4. Gasser, U., Schofield, A., and Weitz, D. A., J. Phys.: Condens. Matter 15, 375-380 (2003).

5. Perera, D. N., and Harrowell, P., Phys. Rev. E 59, 5721-5743 (1999).

6. Binder, K., Baschnagel, J., Kob, W., and Paul, W., "Simulation of models for the glass transition: Is there progress?", in Bridging the time scales: molecular simulations for the next decade, edited by Nielaba, P., Mareschal, M., and Ciccotti, G., Springer-Verlag, Berlin, 2002, pp. 199-228.

7. Donati, C., Glotzer, S. C., Poole, P. H., Kob, W., and Plimpton, S. J., Phys. Rev. E 60, 3107-3119 (1999).

8. Similar binary 2D mixtures were used earlier to investigate hydrodynamic interactions in the liquid state far away from the glass transition: Kollmann, M., Hund, R., Rinn, B., Nägele, G., Zahn, K., König, H., Maret, G., and Klein, R., Europhys. Lett., 919-925 (2002).

9. Kondo, T., Tsumuraya, K., and Watanabe, M. S., J. Chem. Phys.93, 5182-5186 (1990).

10. Miyagawa, H., Hiwatari, Y., Bernu, B., and Hansen, J. P., J. Chem. Phys. 88, 3879-3886 (1988).

11. König, H., Local particle rearrangements in a twodimensional binary colloidal glass former, in the same issue.

12. König, H., to be published.

13. DYNAL PARTICLES AS, http://www.dynalbiotech.com.

14. Zahn, K., Mèndez-Alcaraz, J. M., and Maret, G., Phys. Rev. Lett. 79, 175-178 (1997).

15. Zahn, K., unpublished.

16. Wille, A., Zahn, K., Valmont, F., and Maret, G., Europhys. Lett. 57, 219-225 (2000).

17. Wen, W., Zhang, L., and Shen, P., Phys. Rev. Lett. 85, 5464-5467 (2000). 